\title{
$Q$-deformed description of excitons and associated physical results
}

\author{
M. Bagheri Harouni, R. Roknizadeh, M. H. Naderi \\ Quantum Optics Group, Physics Department, University of Isfahan, Iran \\ E-mail: m-baghreri@phys.ui.ac.ir, rokni@sci.ui.ac.ir, \\ mhnaderi@phys.ui.ac.ir
}

\begin{abstract}
We consider excitons in a quantum dot as $q$-deformed systems. Interaction of some excitonic systems with one cavity mode is considered. Dynamics of the system is obtained by diagonalizing total Hamiltonian and emission spectrum of quantum dot is derived. Physical consequences of $q$-deformed exciton on emission spectrum of quantum dot is given. It is shown that when the exciton system deviates from Bose statistics, emission spectra will become multi peak. With our investigation we try to find the origin of the $q$-deformation of exciton. The optical response of excitons, which affected by the nonlinear nature of $q$-deformed systems, up to the second order of approximation is calculated and absorption spectra of the system is given.
\end{abstract}

PACS numbers: 73.20.La, 71.35.Cc, 03.65.Fd

Submitted to: J. Phys. B: At. Mol. Phys. 


\section{Introduction}

Exciton is an elementary excitation of a semiconductor which consists of a pair of two correlated fermions, the electron and the hole. Analogous to the Hydrogen atom, it is characterized by a binding energy $E_{b}$ and a Bohr radius $a_{B}$. Because an exciton is composed of two fermions, it is a composite boson. Particularly, in a bulk semiconductor, when the excitation of system is dilute, i.e. $n_{e x} a_{B}^{3} \ll 1$, where $n_{e x}$ is the exciton density, a bosonic description of system is convenient [1]. Also Bose-Einstein condensation of excitons, which is an essential characteristic of boson systems has been considered theoretically [2]. When density of excitons increase, the above condition is violated. In this situation the statistics of excitons deviates from Bose statistics.

In low dimensional semiconductor systems such as quantum well (QW), quantum wire and quantum dot (QD), due to the small dimensions and loss of translational symmetry, exciton excitation differs from exciton in bulk materials. In semiconductor nanostructures the size of the system strongly affects exciton properties. For example, in the case of quantum well it is shown [3], if the well width is larger (smaller) than the Bohr radius of exciton, the spectrum of quantum well has properties similar to the situation in which excitons are boson (fermion). Hence, the size of the system directly affects the quantum statistics of excitons in that system. Recently similar results has been obtained for QD [4]. In Ref. [4] the effects of different statistics of excitons on emission spectra of a QD is investigated, and the origin of different statistics of excitons is considered. The same results have also been obtained for the quantum well. If the size of QD is smaller (larger) than the exciton Bohr radius, excitons behave like fermion (boson). Real statistics of excitons in the interaction is considered in [5] and references therein. As mentioned before in high density regime, exciton statistics deviates from Bose statistics. This is due to the increase of mutual forces between the excitations of the system and then the Pauli exclusion principle plays a dominant role [6]. Appearance of Bose statistics of exciton-biexciton system and Pauli exclusion effects in superlattice has been considered experimentally [7].

Bosons and fermions are the only two kinds of particles realized in nature. The conditions mentioned for excitons (in one regime they are like bosons and in another one like fermions) are property of a special kind of statistics called intermediate statistics [8]. Bose and Fermi statistics are two limiting cases of this statistics. Properties of this statistics have been considered by many authors [9]-[11]. Operator realization of intermediate statistics is similar to $q$-deformed operators [12]. Bosonic $q$-deformed operators [13] are a generalization of the Heisenberg algebra obtained by introducing a deformation parameter $q$. Deviation of this parameter from 1 shows deviation of algebra from the Heisenberg algebra. It is shown that it is possible to describe correlated fermion pairs with $q$-deformed bosons [14]. Therefore it is reasonable to consider an exciton system as a $q$-deformed system. We assume the creation and annihilation operators of excitons obey a $q$-deformed algebra. A $q$-deformed description of Frenkel exciton has been considered recently [15]. 
The algebra generated by $q$-deformed operators are given by

$$
\begin{array}{ll}
{\left[\hat{b}_{q}, \hat{b}_{q}^{\dagger}\right]_{q}=\hat{b}_{q} \hat{b}_{q}^{\dagger}-q^{-1} \hat{b}_{q}^{\dagger} \hat{b}_{q}=q^{\hat{n}},} \\
{\left[\hat{n}, \hat{b}_{q}^{\dagger}\right]=\hat{b}_{q}^{\dagger}, \quad\left[\hat{n}, \hat{b}_{q}\right]=-\hat{b}_{q} .}
\end{array}
$$

where $\hat{n}=\hat{b}^{\dagger} \hat{b}$ is the usual particle number operator. Representation of this algebra is given in [16]. In the case of excitons, $q$-parameter can depend on excitation number and physical size of system.

In this paper we consider the interaction of light with a $\mathrm{QD}$ embedded in a microcavity. By considering excitons in QD as $q$-deformed bosons (case of $q$-deformed fermion is straightforward) we study the emission spectrum of the system. As is clear, the commutator (11) explicitly depends on the number of excitons. Hence, this is a system in which light interacts with a nonlinear active medium. Therefore, we shall obtain the linear and nonlinear response of a $q$-deformed exciton system. Knowledge of interaction of light with a nonlinear medium ( $q$-deformed excitons) and its optical response is important for the interpretation of experimental results such as [30]. On the other hand, we compared the obtained results with some experimental ones and in this manner we investigate the physical origin of $q$-deformation of excitons. In section 2 we derive the spectrum of QD when one exciton mode interacts with a single mode cavity-field. In section 3 we consider the interaction of two exciton modes with a single cavity mode. In section 4 the nonlinear response of QD is derived up to second order of approximation. Finally we summarize our conclusions in section 5.

\section{Model Hamiltonian}

We consider a QD embedded in a microcavity which interacts with a single mode cavityfield. We assume the excitations in QD have an intermediate statistics [4], and their creation and annihilation operators obey $q$-deformed algebra. We can express the $q$ deformed operators in terms of ordinary boson operators by the following maps

$$
\hat{b}_{q}=\hat{b} \sqrt{\frac{q^{\hat{n}}-q^{-\hat{n}}}{\hat{n}\left(q-q^{-1}\right)}}, \quad \hat{b}_{q}^{\dagger}=\sqrt{\frac{q^{\hat{n}}-q^{-\hat{n}}}{\hat{n}\left(q-q^{-1}\right)}} \hat{b}^{\dagger},
$$

where $\hat{b}$ and $\hat{b}^{\dagger}$ are the ordinary boson operators and $\hat{n}=\hat{b}^{\dagger} \hat{b}$. Ordinary commutator of $q$-deformed exciton operators is

$$
\left[\hat{b}_{q}, \hat{b}_{q}^{\dagger}\right]=\frac{q}{q+1}\left[q^{n}+q^{-(n+1)}\right] \equiv k(\hat{n}) .
$$

Deviation of this commutator from ordinary boson algebra $\left(\left[\hat{b}, \hat{b}^{\dagger}\right]=1\right)$ relates to deviation of $q$-parameter from 1 . It is clear, this generalized commutator depends on the number of excitations. It seems that by using this algebra we can consider some nonlinear phenomena in the system related to the population of excitons. For example, biexciton effects can be considered in this manner as an effective approach. So that the deformation parameter $q$ can represent some physical parameters such as the ratio of size 
of system to the Bohr radius of exciton. Interaction of QD with single mode cavity-field in rotating wave approximation can be described by the following Hamiltonian

$$
\hat{H}=\hbar \omega \hat{a}^{\dagger} \hat{a}+\hbar \omega_{e x} \hat{b}_{q}^{\dagger} \hat{b}_{q}+\hbar g\left(\hat{a} \hat{b}_{q}^{\dagger}+\hat{a}^{\dagger} \hat{b}_{q}\right)
$$

where $\hat{a}$ and $\hat{a}^{\dagger}$ are creation and annihilation operators of cavity field and $\left[\hat{a}_{i}, \hat{a}_{j}^{\dagger}\right]=\delta_{i j}$. We shall consider a phenomenological damping for the system which relates to both subsystems: photon and exciton. As is clear from the Hamiltonian (44), the exciton number is not a constant of motion. Because of the dependence of exciton operator, $\hat{b}_{q}$, on the exciton number, resulting equations of motion become a nontrivial set of coupled equations. On the other hand, since the total number of excitation (exciton and photon) is conserved we can diagonalize the Hamiltonian in the subspace of a definite excitation. To consider this dynamics we propose an approach based on diagonalization of the Hamiltonian by using the polariton transformation [17]. This procedure depends on some unitary transformations which diagonalize the model Hamiltonian. As is usual in this procedure [18], new operators have the same commutation relation as the original operators (free operators). Here, there are two distinct sets of operators, the cavity mode operators which obey the usual boson commutation relation and exciton operators that are $q$-deformed boson. Therefore, with the presence of these two different statistics, mixed operators (polariton operators) do not have specific statistics. They can be considered as ordinary boson operators or $q$-deformed operators. We consider both situations and we study the physical results associated with each situation in the resonance fluorescence spectrum of QD.

\subsection{Boson polaritons}

In order to solve the dynamical system, we perform the following transformation

$$
\hat{p}_{k}=u_{k} \hat{b}_{q}+v_{k} \hat{a} \text {. }
$$

Due to the presence of $q$-deformed operator $\hat{b}_{q}$, we call this transformation a polaritonlike transformation. As mentioned before, $\hat{b}_{q}$ depends on the number of excitons explicitly and this causes the Hopfield coefficients $u_{k}$ and $v_{k}$ will depend on the number of excitons. Hence, the transformation (5) can be considered as a nonlinear polariton transformation. This kind of transformation has been considered recently for the case of Bogoliubov transformation [19, 20]. We assume polariton-like operators obey the usual boson commutation relations

$$
\left[\hat{p}_{k}, \hat{p}_{k^{\prime}}^{\dagger}\right]=\delta_{k k^{\prime}} \Rightarrow\left[\hat{p}_{k}, \hat{p}_{k}^{\dagger}\right]=\left|u_{k}\right|^{2} k(\hat{n})+\left|v_{k}\right|^{2}=1,
$$

where the operator valued function $k(\hat{n})$ was introduced by Eq.(3). We choose unknown coefficients $u_{k}$ and $v_{k}$ so that the Hamiltonian (4) becomes diagonal in terms of the polariton-like operators

$$
\hat{H}=\hbar \sum_{k} \Omega_{k} \hat{p}_{k}^{\dagger} \hat{p}_{k}
$$


where $\Omega_{k}$ is the polariton spectrum and $k$ refers to different polariton branches. By taking into account a phenomenological damping for exciton and photon systems separately, the unknown parameters satisfy following set of equations

$$
\left[\omega_{e x} k(\hat{n})-\Omega_{k}-i \gamma_{e x}\right] u_{k}+v_{k} g=0, \quad u_{k} g k(\hat{n})+\left(\omega-\Omega_{k}-i \gamma_{p h}\right) v_{k}=0 .
$$

In this set of equations, $\gamma_{e x}$ and $\gamma_{p h}$ are the exciton and photon damping constants, respectively. From these equations the polariton spectrum can be obtained as

$$
\begin{aligned}
\Omega_{k} & =\frac{\omega_{e x} k(\hat{n})+\omega-i\left(\gamma_{e x}+\gamma_{p h}\right)}{2} \\
& \pm \frac{1}{2} \sqrt{\left.\left[\omega_{e x} k(\hat{n})-\omega-i\left(\gamma_{e x}-\gamma_{p h}\right)\right]^{2}+4 g^{2} k(\hat{n})\right)} .
\end{aligned}
$$

It is apparent that $q$-deformed description of excitons causes the splitting between these energy eigenvalues be increased in compare to the case of bosonic description of exciton. Using the set of equations (8) and the polariton spectrum (9) we find the coefficients for two polariton branches

$$
\begin{aligned}
& u_{k}=\sqrt{\frac{\omega-i \gamma_{p h}-\Omega_{k}}{k(\hat{n})\left[\omega-2 \Omega_{k}+\omega_{e x} k(\hat{n})-i\left(\gamma_{e x}+\gamma_{p h}\right)\right]}}, \\
& v_{k}=-\sqrt{\frac{\omega_{e x} k(\hat{n})-i \gamma_{e x}-\Omega_{k}}{\omega-2 \Omega_{k}+\omega_{e x} k(\hat{n})-i\left(\gamma_{e x}+\gamma_{p h}\right)}} .
\end{aligned}
$$

By employing these coefficients all necessary parameters for the polariton Hamiltonian are determined.

Now we can consider the dynamics of polariton operators. The time evolution of polariton operators is governed by the polariton Hamiltonian (7)

$$
\hat{\dot{p}}_{k}=\frac{-i}{\hbar}\left[\hat{p}_{k}, \hat{H}\right]=-i \Omega_{k} \hat{p}_{k} .
$$

Let us consider damping effects by taking into account a phenomenological damping term and noise operator in the dynamical equations of polariton operators. Hence, the time evolution of polariton operator is given by

$$
\hat{\dot{p}}_{k}=-i \Omega_{k} \hat{p}_{k}-\Gamma_{k} \hat{p}_{k}+\hat{F}_{\hat{p}_{k}}(t),
$$

where $\hat{F}_{\hat{p}_{k}}(t)$ is the Langevin noise operator which depends on the reservoir variables and $\Gamma_{k}$ is the damping constant of $k$ th polariton branch given by $\Gamma_{k}=\frac{\gamma_{e x}+\gamma_{p h}}{2}$. Correlation functions of the noise operators determine physical properties of the system. The Langevin noise operator are such that their expectation values $\left\langle\hat{F}_{x}\right\rangle$ vanishes, but their second order moments do not [21]. They are intimately linked up with the global dissipation and in a Markovian environment they take the form

$$
\left\langle\hat{F}_{\hat{p}_{k}}^{\dagger}(t) \hat{F}_{\hat{p}_{k}}\left(t^{\prime}\right)\right\rangle=2 \Gamma_{k} \delta\left(t-t^{\prime}\right) .
$$

With neglecting the phonon effects by decreasing the temperature, other sources of damping like spontaneous recombination of exciton and photon loss are considered as Markovian procedures. It follows, on solving Eq.(12), that

$$
\hat{p}_{k}(t)=\hat{p}_{k}(0) e^{\left(-i \Omega_{k}-\Gamma_{k}\right) t}+\int_{0}^{t} e^{\left(-i \Omega_{k}-\Gamma_{k}\right)\left(t-t^{\prime}\right)} \hat{F}_{\hat{p}_{k}}\left(t^{\prime}\right) d t^{\prime} .
$$


In this equation we set initial time equal zero.

The power spectrum of the scattered light for statistical stationary fields is given by 22

$$
S(r, \omega)=\frac{1}{\pi} \operatorname{Re} \int_{0}^{\infty}\left\langle\hat{E}^{-}(r, t) \hat{E}^{+}(r, t+\tau)\right\rangle e^{i \omega \tau} d \tau
$$

where $\hat{E}^{ \pm}$are the positive and negative frequency parts of the electric field operator. Expressing field operators in terms of creation and annihilation operators we have

$$
S(r, \omega)=\frac{A(r)}{\pi} \operatorname{Re} \int_{0}^{\infty}\left\langle\hat{a}^{\dagger}(0) \hat{a}(\tau)\right\rangle e^{i \omega \tau} d \tau
$$

Here, we set $t=0$, and $A(r)$ depends on mode function of the cavity-field.

Now we can express, the field and exciton creation and annihilation operators in terms of polariton ones:

$$
\hat{a}=v_{1}^{*} \hat{p}_{1}+v_{2}^{*} \hat{p}_{2}, \quad \hat{b}_{q}=k(\hat{n})\left(u_{1}^{*} \hat{p}_{1}+u_{2}^{*} \hat{p}_{2}\right),
$$

and at the time $t$ we have

$$
\hat{a}(t)=v_{1}^{*} \hat{p}_{1}(t)+v_{2}^{*} \hat{p}_{2}(t) .
$$

Now to calculate the resonance fluorescence spectrum we have to determine the initial state of system. we assume at $t=0$, the cavity-field is in a coherent state $|\alpha\rangle$, and the exciton subsystem in its vacuum state. Under this condition, by using Eq.(14) the resonance fluorescence spectrum is obtained as

$$
S(r, \omega)=\frac{A(r)|\alpha|^{2}}{\pi}\left[\left|v_{1}\right|^{2} \frac{\Gamma_{1}}{\left(\omega-\Omega_{1}\right)^{2}+\Gamma_{1}^{2}}+\left|v_{2}\right|^{2} \frac{\Gamma_{2}}{\left(\omega-\Omega_{2}\right)^{2}+\Gamma_{2}^{2}}\right] .
$$

In deriving this result we implicitly assume that at $t=0$ the noise operator and polariton operators are uncorrelated. Fig.(1) shows the plot of $S(r, \omega)$ versus $\omega$ for different values of deformation parameter q. Material parameters are chosen as $\omega=1.75 \mathrm{eV}$, $\omega_{e x}=1.75 \mathrm{eV}, g=200 \mu \mathrm{eV}, \gamma_{e x}=20 \mu \mathrm{eV}, \gamma_{p h}=40 \mu \mathrm{eV}[23], n=100$ and $|\alpha|^{2}=9$. As is clear when $q=1$, spectrum has similar variation as experimental results [23]. This figure shows that when $q=1$ (nondeformed case) the power spectrum of the fluorescence light is a double peak centered at $\omega=\Omega_{1}$ and $\omega=\Omega_{2}$. By increasing deviation of $q$ from 1 , it is apparent from the different plots in this figure that splitting between two peaks increases and the height of one of peaks decreases. This result has been reported in resonance fluorescence of excitons when the biexcitonic interaction is taken into account. It has been shown [3] that biexcitonic effects are a red shift of the transition frequencies, emergence of sidebands due to the switch-on forbidden transitions and asymmetry of the emission spectrum. The binding energy of biexciton in QD causes a shift in the spectrum of the system. In the present model the splitting of spectrum (Rabi splitting) depends on the $q$-parameter. Hence, changing this parameter affects the spectrum. Then as a one reason of deviation of excitons from ideal Bose system we can consider Coulomb interaction between them. On the other hand, $q$-deformed exciton operators depend on the total number of exciton, and biexciton interaction occurs when there are more than one exciton. This similarity makes this clue that the $q$-deformation can be consider as 
an effective approach to take into account the biexciton effects. As mentioned before, the $q$-parameter can depend on the size of sample. The plotted resonance fluorescence spectrum in Fig.(11) makes clear some differences of optical properties of different size QD. For large values of q, compare with 1, spectrum will reduce to one peak. This case is a characteristic of the weak coupling regime.

\subsection{Q-deformed polaritons}

In this subsection we assume that the polariton operators are $q$-deformed operators. According to the $q$-deformed nature of the exciton system we assume the following algebra for polariton operators

$$
\left[\hat{p}_{k}, \hat{p}_{k}^{\dagger}\right]_{s}=\hat{p}_{k} \hat{p}_{k}^{\dagger}-s^{-1} \hat{p}_{k}^{\dagger} \hat{p}_{k}=s^{\hat{n}_{k}}
$$

where $s$ denotes the deformation parameter corresponding to the polariton system and $\hat{n}_{k}$ shows the number operator for $k$ th polariton branch. Ordinary commutator for these operators is

$$
\left[\hat{p}_{k}, \hat{p}_{k}^{\dagger}\right]=\left|u_{k}\right|^{2} k(\hat{n})+\left|v_{k}\right|^{2}=\frac{s}{s+1}\left[s^{\hat{n}_{k}}+s^{-\left(\hat{n}_{k}+1\right)}\right]=M\left(\hat{n}_{k}\right) .
$$

Using the same approach of the previous subsection we obtain the following set of equations for the coefficients of transformation

$$
\begin{aligned}
& {\left[\left(\omega_{e x} k(\hat{n})-i \gamma_{e x}-\Omega_{k}^{\prime} M\left(n_{k}\right)\right] u_{k}+v_{k} g=0,\right.} \\
& u_{k} g k(\hat{n})+\left[\omega-i \gamma_{p h}-\Omega_{k}^{\prime} M\left(n_{k}\right)\right] v_{k}=0 .
\end{aligned}
$$

From this set of equations we derive the deformed polariton spectrum as

$$
\begin{aligned}
\Omega_{k}^{\prime} & =\frac{\omega_{e x} k(\hat{n})+\omega-i\left(\gamma_{e x}+\gamma_{p h}\right)}{2 M\left(n_{k}\right)} \\
& \pm \frac{\sqrt{\left.\left[\omega_{e x} k(\hat{n})-\omega-i\left(\gamma_{e x}-\gamma_{p h}\right)\right]^{2}+4 g^{2} k(\hat{n})\right)}}{2 M\left(n_{k}\right)}
\end{aligned}
$$

and the transformation coefficients read as

$$
\begin{aligned}
& u_{k}=-\sqrt{\frac{M\left(n_{k}\right)\left[\omega-i \gamma_{p h}-\Omega_{k}^{\prime} M\left(n_{k}\right)\right]}{k(\hat{n})\left[\omega-2 \Omega_{k}^{\prime} M\left(n_{k}\right)+\omega_{e x} k(\hat{n})-i\left(\gamma_{e x}+\gamma_{p h}\right)\right]}}, \\
& v_{k}=\sqrt{\frac{M\left(n_{k}\right)\left[\omega_{e x} k(\hat{n})-i \gamma_{e x}-\Omega_{k}^{\prime} M\left(n_{k}\right)\right]}{\omega-2 \Omega_{k}^{\prime} M\left(n_{k}\right)+\omega_{e x} k(\hat{n})-i\left(\gamma_{e x}+\gamma_{p h}\right)}} .
\end{aligned}
$$

By determining all the variables, polariton Hamiltonian (diagonal Hamiltonian) will be determined. By applying the same procedure as before we derive the resonance fluorescence spectrum in this case as follows

$$
S(r, \omega)=\frac{A(r)|\alpha|^{2}\left(\left|v_{1}\right|^{2}+\left|v_{2}\right|^{2}\right)}{\pi} \sum_{i=1,2}\left|v_{i}\right|^{2} \frac{\Gamma_{i}}{\left(\omega-\Omega_{i}^{\prime} M\left(n_{k}\right)\right)^{2}+\Gamma_{i}^{2}} .
$$

Fig. (2) shows the plot of $S(r, \omega)$ versus $\omega$ for different values of polariton deformation parameter $s$. This figure shows that changes of s-parameter (deformation parameter of polariton) does not cause any shift in transition frequencies, but causes strengths of peaks increase. 


\section{Interaction of light with two exciton modes}

We now consider the interaction of one cavity mode with QD when two exciton modes are coupled to the field mode. As before, we assume exciton system is expressed by the $q$-deformed operators. The total Hamiltonian of the system under consideration can be written as follows

$$
\hat{H}=\hbar \omega \hat{a}^{\dagger} \hat{a}+\hbar \sum_{i=1,2} \omega_{e x_{i}} \hat{b}_{q_{i}}^{\dagger} \hat{b}_{q_{i}}+\hbar g \sum_{i=1,2}\left(\hat{a} \hat{b}_{q_{i}}^{\dagger}+\hat{a}^{\dagger} \hat{b}_{q_{i}}\right) .
$$

We assume both excitons have the same coupling constant with the cavity mode. We solve this system as before by diagonalizing the Hamiltonian. For this purpose we perform the following transformation

$$
\hat{p}_{k}=u_{k} \hat{b}_{q_{1}}+x_{k} \hat{b}_{q_{2}}+v_{k} \hat{a} .
$$

We consider the situation in which the polariton operators obey the nondeformed Bose statistics

$$
\left[\hat{p}_{k}, \hat{p}_{k}^{\dagger}\right]=\left|u_{k}\right|^{2} k\left(\hat{n}_{1}\right)+\left|x_{k}\right|^{2} k\left(\hat{n}_{2}\right)+\left|v_{1}\right|^{2}=1,
$$

where $\hat{n}_{i}(i=1,2)$ represents the number operator for each excitonic mode. As is clear in this case there are three polariton branches. Assuming the transformation (27) diagonalizes the Hamiltonian (26), this polariton Hamiltonian takes the following form

$$
\hat{H}=\hbar \sum_{k} \Omega_{k} \hat{p}_{k}^{\dagger} \hat{p}_{k}
$$

where summation is over all polariton branches. The following equation determines the polariton spectrum

$$
\left(c-\Omega_{k}\right)\left[\left(d-\Omega_{k}\right)\left(\omega-i \gamma_{p h}-\Omega_{k}\right)-g^{2} k\left(\hat{n}_{1}\right)\right]-g^{2} k\left(\hat{n}_{2}\right)\left(d-\Omega_{k}\right)=0,
$$

where $c=\omega_{e x_{1}} k\left(\hat{n}_{1}\right)-i \gamma_{e x_{1}}$ and $d=\omega_{e x_{2}} k\left(\hat{n}_{2}\right)-i \gamma_{e x_{2}}$. By deriving the polariton spectrum the transformation parameters are obtained as

$$
\begin{aligned}
& u_{k}=\frac{g\left[\left(d-\Omega_{k}\right)\left(\omega-i \gamma_{p h}-\Omega_{k}\right)-g^{2} k\left(\hat{n}_{2}\right)\right]}{A}, \\
& x_{k}=\frac{g^{3} k\left(\hat{n}_{1}\right)}{A} \\
& v_{k}=-\frac{\left(c-\Omega_{k}\right)\left[\left(d-\Omega_{k}\right)\left(\omega-i \gamma_{p h}-\Omega_{k}\right)-g^{2} k\left(\hat{n}_{2}\right)\right]}{A},
\end{aligned}
$$

where the parameter $A$ is given by

$$
\begin{aligned}
A & =\left(\left[g^{2} k\left(\hat{n}_{1}\right)+\left(c-\Omega_{k}\right)^{2}\right]\left[\left(d-\Omega_{k}\right)\left(\omega-i \gamma_{p h}-\Omega_{k}\right)-g^{2} k\left(\hat{n}_{2}\right)\right]^{2}\right. \\
& \left.+g^{6} k^{2}\left(\hat{n}_{1}\right) k\left(\hat{n}_{2}\right)\right)^{\frac{1}{2}} .
\end{aligned}
$$

In this manner, all the parameters which appear in the polariton Hamiltonian are determined. By repeating the approach of previous section the resonance fluorescence spectrum of system with different initial conditions can be determined. If we assume 
at $t=0$ the cavity mode is in the coherent state $|\alpha\rangle$ and $\mathrm{QD}$ in vacuum state $|0\rangle$, the resonance fluorescence spectrum is given by

$$
S(r, \omega)=\frac{|\alpha|^{2} A(r)}{\pi} \sum_{k} \frac{\left|v_{k}\right|^{2} \Gamma_{k}}{\Gamma_{k}^{2}+\left(\omega-\Omega_{k}\right)^{2}} .
$$

To show complex structure (multi-peak structure) of this spectrum Fig. (3) presents the spectra on a logarithmic scale. For the sake of clarity, we have powered some peaks compare to other ones in this figure. In the case of $q=1$ (nondeformed exciton) the spectrum has two peaks. Increasing the $q$-parameter causes that splitting between peaks be increased and spectrum becomes multi-peaks. Multi-peaks structure in emission of exciton such as Mollow triplet was predicted when excitons obey statistics different from Bose statistics [3, 4]. When, $q$-parameter is changed, the energy and intensities of emission change. Effects of exciton number on absorption spectrum of QD is considered . Due to the relation of absorption spectrum and resonance fluorescence, similar result is obtain in [24].

\section{Nonlinear response of excitons in $q$-deformed regime}

In previous sections we considered some physical results of $q$-deformed description of excitons. The $q$-deformed description can be served as a nonlinear description of excitons. It is well-known that different kinds of nonlinearity in an exciton system lead to different orders of nonlinear response of the system [25, 26]. Therefore, we try to obtain optical response of a driven quantum dot, which its optical excitations are considered as $q$-deformed systems. For this purpose we will calculate the coefficient absorption of a QD in this regime. In this section we neglect all damping effects and we consider the Hamiltonian of the system as follows

$$
\hat{H}=\hbar \omega \hat{a}^{\dagger} \hat{a}+\hbar \omega_{e x} \hat{b}_{q}^{\dagger} \hat{b}_{q}+\hbar g\left(\hat{a} \hat{b}_{q}^{\dagger}+\hat{a}^{\dagger} \hat{b}_{q}\right)
$$

In the electron picture, the induced dipole moment by transition of an electron is described by $\hat{\mu}=\hat{a}_{v}^{\dagger} \hat{a}_{c}+\hat{a}_{c}^{\dagger} \hat{a}_{v}$ [27]. The operator $\hat{a}_{v}^{\dagger}\left(\hat{a}_{v}\right)$ is the creation (annihilation) operator for an electron in the valance band (level in the case of QD), and $\hat{a}_{c}^{\dagger}\left(\hat{a}_{c}\right)$ is the creation (annihilation) operator for an electron in the conduction band. Hence, creation of an exciton is denoted by $\hat{a}_{c}^{\dagger} \hat{a}_{v}=\hat{b}_{q}^{\dagger}$. Therefore we can write the dipole operator of $\mathrm{QD}$ as $\hat{\mu}=\hat{b}_{q}^{\dagger}+\hat{b}_{q}$. The macroscopic polarization is expectation value of polarization operator. The optical response function represents the reaction of the system to an external classic field $E(t)$ coupled to the variables of system [28], i.g., the dipole operator. Hence, we consider an external field as a pump source and we treat the reaction of QD to it. Then the total Hamiltonian of system is then given by

$$
\hat{H}=\hbar \omega \hat{a}^{\dagger} \hat{a}+\hbar \omega_{e x} \hat{b}_{q}^{\dagger} \hat{b}_{q}+\hbar g\left(\hat{a} \hat{b}_{q}^{\dagger}+\hat{a}^{\dagger} \hat{b}_{q}\right)-\left[\vec{d}_{v c} \cdot \vec{E}(t) \hat{b}_{q}+\vec{d}_{c v} \cdot \vec{E}(t) \hat{b}_{q}^{\dagger}\right],
$$

where $\vec{d}_{v c}$ denotes the dipole matrix element. The Hamiltonian in the interaction picture has the form

$$
\hat{H}_{\text {int }}=\hbar g\left[\hat{a} \hat{b}_{q}^{\dagger} e^{-i\left[\omega-\omega_{e x} k(\hat{n})\right] t}+\hat{a}^{\dagger} e^{i\left[\omega-\omega_{e x} k(\hat{n})\right] t} \hat{b}_{q}\right]
$$




$$
-\left[\vec{d}_{c v} \cdot \vec{E}(t) e^{-i \omega_{e x} k(\hat{n}) t} \hat{b}_{q}+\vec{d}_{v c} \cdot \vec{E}(t) \hat{b}_{q}^{\dagger} e^{i \omega_{e x} k(\hat{n}) t}\right] .
$$

The observable of interest for the optical response is the time-dependent dipole density $\mu(t)=\left\langle\hat{b}_{q}(t)\right\rangle+$ h.c. $=\operatorname{Tr}_{e x}\left(\hat{b}_{q} \rho_{e x}(t)\right)+$ h.c. , where $\operatorname{Tr}_{e x}$ means trace over the exciton system and $\rho_{e x}(t)=\operatorname{Tr}_{f} \rho(t)$, which $\rho(t)$ is the total time dependent density matrix of the system and $\rho_{e x}(t)$ is the time dependent density matrix of exciton system. The total time dependent density matrix is given by

$$
\hat{\rho}(t)=\hat{U}\left(t, t_{0}\right) \hat{\rho}\left(t_{0}\right) \hat{U}^{-1}\left(t, t_{0}\right),
$$

where $U\left(t, t_{0}\right)=\hat{T} \exp \left[-\frac{i}{\hbar} \int_{t_{0}}^{t} \hat{H}_{\text {int }}\left(t^{\prime}\right) d t^{\prime}\right]$ is the time ordered evolution operator and $\hat{\rho}\left(t_{0}\right)$ is the total density matrix of system at initial time. We assume that the quantum field and exciton system are both in vacuum state. Therefore, the time dependent density matrix of excitons is given by

$$
\hat{\rho}_{e x}(t)=\sum_{n}\left\langlen \left|\hat { U } ( t , t _ { 0 } ) ( | 0 \rangle _ { f } | 0 \rangle _ { e x } ) \left(_{e x}\left\langle\left. 0\right|_{f}\langle 0|\right) \hat{U}^{-1}\left(t, t_{0}\right)|n\rangle,\right.\right.\right.
$$

where summation is carried on field state and the matrix elements of the time evolution operator are in the basis of field states. By using the Feynman disentanglement theorem 29] the matrix elements of the time evolution operator $\hat{U}\left(t, t_{0}\right)$ can be evaluated. We can write Hamiltonian in (36) as $\hat{H}_{\text {int }}=\hat{H}_{1}(t)+\hat{H}_{2}(t)$, where

$$
\begin{aligned}
& \hat{H}_{1}(t)=\hbar g\left[\hat{a} \hat{b}_{q}^{\dagger} e^{-i\left[\omega-\omega_{e x} k(\hat{n})\right] t}+\hat{a}^{\dagger} e^{i\left[\omega-\omega_{e x} k(\hat{n})\right] t} \hat{b}_{q}\right] \\
& \hat{H}_{2}(t)=-\left[\vec{d}_{c v} \cdot \vec{E}(t) e^{-i \omega_{e x} k(\hat{n}) t} \hat{b}_{q}+\vec{d}_{v c} \cdot \vec{E}(t) \hat{b}_{q}^{\dagger} e^{i \omega_{e x} k(\hat{n}) t}\right] .
\end{aligned}
$$

As is clear $\hat{H}_{2}(t)$ depends only on exciton operators. The time evolution operator can be written as

$$
\begin{aligned}
\hat{U}\left(t, t_{0}\right) & =\hat{T} \exp \left[-\frac{i}{\hbar} \int_{t_{0}}^{t}\left(\hat{H}_{1}\left(t^{\prime}\right)+\hat{H}_{2}(t)\right) d t^{\prime}\right] \\
& =\hat{T} \exp \left[-\frac{i}{\hbar} \int_{t_{0}}^{t} \hat{H}_{2}\left(t^{\prime}\right) d t^{\prime}\right] \exp \left[-\frac{i}{\hbar} \int_{t_{0}}^{t} \hat{H}_{1}(s) d s\right] .
\end{aligned}
$$

In this equation we use Feynman notation [29]. These two exponential terms are not disentangle from each other. They are correlated and in doing integration, we have to take into account ordering of operators. In calculation of matrix element of this operator in the basis of field states, second exponential can be considered as a ordinary c-number function of $t^{\prime}$, because it is independent of field operators:

$$
\left\langle i\left|\hat{U}\left(t, t_{0}\right)\right| j\right\rangle=\left\langle i\left|\hat{T} \exp \left[-\frac{i}{\hbar} \int_{t_{0}}^{t} \hat{H}_{1}\left(t^{\prime}\right) d t^{\prime}\right]\right| j\right\rangle \exp \left[-\frac{i}{\hbar} \int_{t_{0}}^{t} \hat{H}_{2}(s) d s\right] .
$$

On the other hand we consider all the exciton operators in $\hat{H}_{1}(t)$ as ordinary c-number functions, and we can write

$$
\begin{aligned}
\left\langle i\left|\hat{U}\left(t, t_{0}\right)\right| j\right\rangle & =\left\langle i\left|\hat{T} \exp \left[-\frac{i}{\hbar} \int_{t_{0}}^{t}\left(\hat{a}_{t^{\prime}}^{\dagger} e^{i \omega t^{\prime}} B\left(t^{\prime}\right)+\hat{a}_{t^{\prime}} e^{-i \omega t^{\prime}} B^{*}\left(t^{\prime}\right)\right) d t^{\prime}\right]\right| j\right\rangle \\
& \times \exp \left[-\frac{i}{\hbar} \int_{t_{0}}^{t} \hat{H}_{2}(s) d s\right],
\end{aligned}
$$


where $B(t)$ is a ordinary function corresponding to exciton operators. As is clear this matrix element is a function of exciton operators. The influence of exciton system is completely contained in this operator functional and factored term in (42). By using Feynman theorem, above matrix element can written as

$$
\left\langle i\left|\exp \left[-i g \int_{t_{0}}^{t} \hat{a}_{t^{\prime}}^{\dagger} e^{i \omega t^{\prime}} B\left(t^{\prime}\right) d t^{\prime}\right] \exp \left[-i g \int_{t_{0}}^{t} \hat{a}_{t^{\prime}}^{\prime} e^{-i \omega t^{\prime}} B^{*}\left(t^{\prime}\right) d t^{\prime}\right]\right| j\right\rangle,
$$

where in this equation $\hat{a}_{t^{\prime}}^{\prime}=\hat{V}^{-1}(t) \hat{a}_{t} \hat{V}(t)$, and

$$
\hat{V}(t)=\exp \left[-i g \hat{a}^{\dagger} \int_{t_{0}}^{t} B\left(t^{\prime}\right) e^{i \omega t^{\prime}}\right] .
$$

In this manner the density matrix of exciton system takes the following form

$$
\hat{\rho}_{e x}(t)=\sum_{n} \frac{1}{n !} S_{1}\left(\hat{n}, \hat{b}_{q}, \hat{b}_{q}^{\dagger}\right)|0\rangle_{e x}\langle 0| S_{2}\left(\hat{n}, \hat{b}_{q}, \hat{b}_{q}^{\dagger}\right),
$$

where

$$
\begin{aligned}
S_{1}\left(\hat{n}, \hat{b}_{q}, \hat{b}_{q}^{\dagger}\right) & =\left[-g \hat{b}_{q}^{\dagger} \frac{e^{i \omega_{e x} k\left(\hat{n}_{e x}\right)\left(t-t_{0}\right)}}{\omega_{e x} k\left(\hat{n}_{e x}\right)}\right]^{n} e^{-\frac{g^{2}}{2} L\left(\hat{n}_{e x}\right)} f\left(\hat{b}_{q}, \hat{b}_{q}^{\dagger}\right), \\
S_{2}\left(\hat{n}, \hat{b}_{q}, \hat{b}_{q}^{\dagger}\right) & =\left[-g \hat{b}_{q} \frac{e^{-i \omega_{e x} k\left(\hat{n}_{e x}+1\right)\left(t-t_{0}\right)}}{\omega_{e x} k\left(\hat{n}_{e x}+1\right)}\right]^{n} e^{-\frac{g^{2}}{2} L\left(\hat{n}_{e x}\right)} f^{-1}\left(\hat{b}_{q}, \hat{b}_{q}^{\dagger}\right), \\
L\left(\hat{n}_{e x}\right) & =\hat{b}_{q}^{\dagger} \hat{b}_{q}\left[\frac{e^{-i \omega_{e x}\left[k\left(\hat{n}_{e x}+1\right)-k\left(\hat{n}_{e x}-1\right)\right]\left(t-t_{0}\right)}}{\left[\omega-\omega_{e x} k\left(\hat{n}_{e x}-1\right)\right]\left[\omega-\omega_{e x} k\left(\hat{n}_{e x}+1\right)\right]}\right. \\
& \left.+\frac{e^{\left.-i \omega_{e x}\left[k\left(\hat{n}_{e x}+2\right)-k\left(\hat{n}_{e x}\right)\right)\right]\left(t-t_{0}\right)}}{\left[\omega-\omega_{e x} k\left(\hat{n}_{e x}+2\right)\right]\left[\omega-\omega_{e x} k\left(\hat{n}_{e x}\right)\right]}\right]
\end{aligned}
$$

and

$$
\begin{aligned}
f\left(\hat{b}_{q}, \hat{b}_{q}^{\dagger}\right)=\hat{T} \exp [ & \frac{i}{\hbar} \int_{t_{0}}^{t} d t^{\prime}\left(\vec{d}_{c v} \cdot \vec{E}\left(t^{\prime}\right) \hat{b}_{q} e^{-i \omega_{e x} k\left(\hat{n}_{e x}+1\right) t^{\prime}}\right. \\
& \left.\left.+\vec{d}_{v c} \cdot \vec{E}\left(t^{\prime}\right) \hat{b}_{q}^{\dagger} e^{i \omega_{e x} k\left(\hat{n}_{e x}\right) t^{\prime}}\right)\right]
\end{aligned}
$$

By expanding the function $f\left(\hat{b}_{q}, \hat{b}_{q}^{\dagger}\right)$ up to second order in $E(t)$ and using (45) we obtain the time-dependent dipole density as follows

$$
\begin{aligned}
& p(t)=\sum_{n} \frac{g^{2 n}}{n !} h_{1}(n) ! \sqrt{f_{q}(n) !} e^{-\frac{g^{2}}{2} L(n)} \times \\
& {\left[\frac{i}{\hbar} h_{0}(n+1) ! e^{-\frac{g^{2}}{2} L(1)} \sqrt{f_{q}(n+1) ! f_{q}(n+1)} \int_{t_{0}}^{t} \vec{d}_{c v} \cdot \vec{E}\left(t^{\prime}\right) e^{i \omega_{e x} t^{\prime}} d t^{\prime}\right.} \\
& -\frac{i}{\hbar} \sqrt{f_{q}(n)} h_{0}(n) ! e^{-\frac{g^{2}}{2} L(0)} \sqrt{f_{q}(n) ! f_{q}(n)} \int_{t_{0}}^{t} \vec{d}_{c v} \cdot \vec{E}\left(t^{\prime}\right) e^{i \omega_{e x} k(n-1) t^{\prime}} d t^{\prime} \\
& +\frac{i}{2 \hbar^{3}} \sqrt{f_{q}(n+1)} h_{0}(n+2) ! e^{-\frac{g^{2}}{2} L(0)} \sqrt{f_{q}(n+2) ! f_{q}(n+2) \times} \\
& \int_{t_{0}}^{t} \int_{t_{0}}^{t} \int_{t_{0}}^{t} \vec{d}_{v c} \cdot \vec{E}\left(t^{\prime}\right) \vec{d}_{c v} \cdot \vec{E}(r) \vec{d}_{c v} \cdot \vec{E}(s) e^{i \omega_{e x}\left[s+r-k(n+1) t^{\prime}\right]} d t^{\prime} d r d s \\
& +\frac{i}{2 \hbar^{3}} \sqrt{f_{q}(n)} h_{0}(n) ! e^{-\frac{g^{2}}{2} L(0)} \sqrt{f_{q}(n) ! f_{q}(n)} \times
\end{aligned}
$$




$$
\begin{aligned}
& \int_{t_{0}}^{t} \int_{t_{0}}^{t} \int_{t_{0}}^{t} \vec{d}_{c v} \cdot \vec{E}\left(t^{\prime}\right) \vec{d}_{v c} \cdot \vec{E}(r) \vec{d}_{c v} \cdot \vec{E}(s) e^{i \omega_{e x}\left[k(n-1) t^{\prime}-(r-s)\right]} d t^{\prime} d r d s \\
& -\frac{i}{2 \hbar^{3}} \sqrt{f_{q}(n+1)} h_{0}(n+1) ! e^{-\frac{g^{2}}{2} L(1)} \sqrt{f_{q}(n+1) ! f_{q}(n+1)} \int_{t_{0}}^{t} \int_{t_{0}}^{t} \int_{t_{0}}^{t} \times \\
& \vec{d}_{v c} \cdot \vec{E}(r) \vec{d}_{c v} \cdot \vec{E}(s) \vec{d}_{c v} \cdot \vec{E}\left(t^{\prime}\right) e^{-i \omega_{e x}\left[k(n+1)(r-s)-t^{\prime}\right]} d t^{\prime} d r d s \\
& -\frac{i}{2 \hbar^{3}} \sqrt{f_{q}(n)} h_{0}(n+1) ! e^{-\frac{g^{2}}{2} L(1)} \sqrt{f_{q}(n+1) ! f_{q}(n+1)} \int_{t_{0}}^{t} \int_{t_{0}}^{t} \int_{t_{0}}^{t} \times \\
& \left.\vec{d}_{c v} \cdot \vec{E}(r) \vec{d}_{v c} \cdot \vec{E}(s) \vec{d}_{c v} \cdot \vec{E}\left(t^{\prime}\right) e^{i \omega_{e x}\left[k(n+1)(r-s)+t^{\prime}\right]} d t^{\prime} d r d s\right],
\end{aligned}
$$

where $h_{i}(n)=\frac{e^{(-1)^{i} i \omega_{e x} k(n+i)\left(t-t_{0}\right)}}{\omega_{e x} k(n+i)}$ and $f_{q}(n)=\sqrt{\frac{q_{n}-q^{-n}}{q-q^{-1}}}$. These equation shows that in this conditions second order response function is equal zero. Now we can calculate linear and nonlinear electric susceptibility of this exciton system from this equation. Generalized linear and nonlinear absorption spectra of this system is shown in figures (44)-(6) for different values of $q$-parameter. In these plots, $1 s$-exciton is considered. In these figures we choose $\hbar=e=1, g=200 \mu \mathrm{ev}$ and $\omega_{e x}=1574 \mathrm{mev}$. Fig.(4) shows plots of linear absorption spectra and Fig.(6) shows plots of nonlinear spectra. On the other hand, 3-dimension plot of linear absorption coefficients is given in figure (5). It is clear that changes of $q$-parameter strongly affects absorption spectra of the system. These figures show in the presence of $q$-values absorption of probe beam shows a complex structure: a multiple-like absorption pattern appears with one strong peak and some side bands. Presence of these side bands is a signature of the optical generation of an nonlinear exciton (an exciton which expresses with $q$-deformed operator). Negative part of the absorption spectrum demonstrates gain of the probe beam. Due to the resonance interaction of pump with exciton transition, the gain effect comes from the coherent energy exchange between the pump and probe beams through the QD nonlinearity. The obtained absorption spectra are very similar to experimental results [30]. In Ref. [30] absorption spectra of a driven charged QD is derived experimentally. Charged $\mathrm{QD}$ is a nonlinear medium and is similar to our model. Then It can be consider as a experimental test of our model.

\section{Conclusion}

$Q$-deformed description of excitons in a QD and its physical consequences was considered. We showed that increasing the $q$-parameter will lead to increase of splitting between peaks in the spectrum and asymmetry of spectrum. Similar effects were observe when biexciton effects taken into account. In experiments of QD it is shown [23] the same results are obtained in different temperatures. Then we can associate this physical parameter as source of $q$-deformation. The temperature dependence of emission energy of system can be attributed to the change in the refractive index of its active medium with temperature. We have derived the optical response of QD with $q$-deformed exciton. As mentioned before $q$-deformed description of excitons will lead to dependence of optical response on $q$ parameter. Hence, due to the wide range of $q$ parameter and its effects 
on optical response we can consider some parameters like temperature and interaction between the excitons which affects the optical response of QD as sources of $q$-deformation of excitons. As mentioned, the relation of quantum statistics of excitons in the QD and the size of QD has been considered. Then we can consider the ratio of exciton Bohr radius to dimension of system and exciton population as two main sources of $q$ deformation. $Q$-deformed operator depends on total number of associated particles of system. Therefore we can interpret $q$-deformed operator as an operator which consists of effects of other excitations of system implicitly. Then it is reasonable to consider this description as an effective description which takes into account some nonlinearity in exciton system. As we saw, in the case of interaction of light with two excitons, when $q=1$ this system showed a two peaks spectrum. While by increasing deviation of exciton from Bose statistics, spectrum becomes multi peak. Due to the nonlinear nature of $q$-deformed exciton we showed that different orders of nonlinear response function of this system can be calculated. From coincidence of obtained results and experimental results, we can conclude that $q$-deformed description of excitons can be a considerable model for excitons. With comparing the obtained results in this paper with experimental ones we can investigate the origin of this description of excitons. As pointed out the ratio of system dimension to the Bohr radius of exciton is one of the sources of deviation of excitons from usual boson. The obtained results are very similar to the effects of the exciton-exciton interaction [3], 31] which is relates to exciton population and biexciton binding energy. On the other hand, it is shown that [1] exciton density is another source of their deviation from ordinary bosons. To sum up we attribute the origin of $q$-deformation of the excitons to their density, their mutual interactions, confinement size and other parameters which cause fluctuation of optical response of the system. $Q$-deformed description of an active medium causes that the optical properties of system depend on the $q$-parameter. Then, it is seem that parameters which can affect optical properties of the active medium (like refractive index) their effects can be considered by this formulation. The $q$-parameter can be considered as a variation parameter which its values can be obtained from comparison of theoretical and experimental results.

Acknowledgment The authors wish to thank the Office of Graduate Studies of the University of Isfahan and Iranian Nanotechnology initiative for their support.

\section{References}

[1] A. S. Davydov, Theory of Molecular Excitons, (Plenum Press, NewYork, 1971).

[2] E. Hanamura and H. Haug, Phys. Rep. 33, 209 (1977).

[3] Y. Yamamoto, F. Tassone and H. Cao, Semiconductor Cavity Electrodynamics, (Springer-Verlag Berlin Heidelberg, 2000).

[4] F. P. Laussy, M. M. Glazov, A. Kavokin, D. M. Whittaker and G. Malpuech, Phys. Rev. B 73, $115343(2006)$.

[5] B. Laikhtman, J. Phys: Condens. Matter 19, 295214 (2007).

[6] M. Combescot, O. Betbeder-Matibet and F. Dubin, Phys. Rev. A 76, 033601 (2007).

[7] H. Ichida, M. Nakayama and J. Lumin, J. Lumin. 94-95, 379 (2001).

[8] A. Khare, Fractional Statistics and Quantum Theory, (World Scientific, Singapore, 1997). 
[9] M. P. Blencowe and N. C-Koshnick, J. Math. Phys. 42, 5713 (2001).

[10] W. S. Dai and M. Xie, Physica A 331, 497 (2204).

[11] Y. Shen, W. S. Dai and M. Xie, Phys. Rev. A 75, 042111(2007).

[12] S. Chaturvedi, V. Srinivasan, Phys. Rev. A 44, 8024 (1991).

[13] A. J. Macfarlane, J. Phys. A: Math. Gen. 22, 4581 (1989); L. C. Biedenharn, J. Phys. A: Math. Gen. 22, L873 (1989).

[14] D. Bonatsos, J. Phys. A: Math. Gen. 25, L101 (1992).

[15] Y. X. Liu, C. P. Sun, S. X. Yu and D. L. Zhou, Phys. Rev. A 63, 023802 (2001).

[16] G. Rideau, Lett. Math. Phys. 24, 147(1992).

[17] J. J. Hopfield, Phys. Rev. 112, 1555 (1958).

[18] U. Fano. Phys. Rev. 103, 1202 (1956).

[19] J. Katriel, Phys. Lett. A 307, 1(2003).

[20] M. H. Naderi, R. Roknizadeh and M. Soltanolkotabi, Prog. Theor. Phys. 112, 797 (2004); ibid 112, $811(2004)$.

[21] M. Lax, Phys. Rev. 145, 110 (1966).

[22] M. O. Scully, M. S. Zubairy, Quantum Optics, (Cambridge University Press 1997).

[23] E. Peter, P. Senellart, D. Martrou, A. Lemaître, J. Hours, J. M. Gérard, and J. Bloch, Phys. Rev. Lett. 95, 067401 (2005).

[24] A. Franceschetti and Y. Zhang, Phys. Rev. Lett. 100, 136805 (2008).

[25] V. M. Axt, A. Stahl, Z. Phys. B 93, 205 (1994).

[26] Th. Östreich, K. Schönhammer, L. J. Sham, Phys. Rev. Lett. 74, 4698 (1995).

[27] H. Haug, S. W. Koch, Quantum Theory of The Optical and Electronic Properties of Semiconductor, 4rd edition (World Scientific, Singapore, 2004).

[28] S. Mukamel, Principles of Nonlinear Optical Spectroscopy, (Oxford, NewYork, 1995).

[29] R. P. Feynman, Phys. Rev. 84, 108 (1951).

[30] X. Xu et al., arxiv:0803,0734 (Cond-Mat. mes-hall).

[31] U. Hohenester and E. Molinari, Phys. Stat. Sol. (b) 221, 19 (2000). 


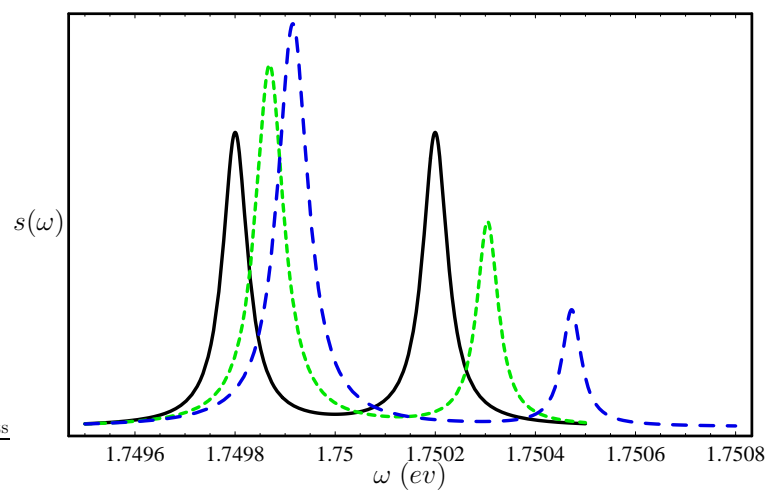

Figure 1. Plots of $S(\omega)$ versus $\omega$. Parameter are choose as $\omega=1.75 \mathrm{eV}$, $\omega_{e x}=1.75 \mathrm{eV}, g=200 \mu \mathrm{eV}, \gamma_{e x}=20 \mu \mathrm{eV}, \gamma_{p h}=40 \mu \mathrm{eV}, n=1$ and $|\alpha|^{2}=9$. Solid plot corresponds to $q=1$, nondeformed case. Dotted one corresponds to $q=1.01$, and for dash line $q$ is equal 1.015 .

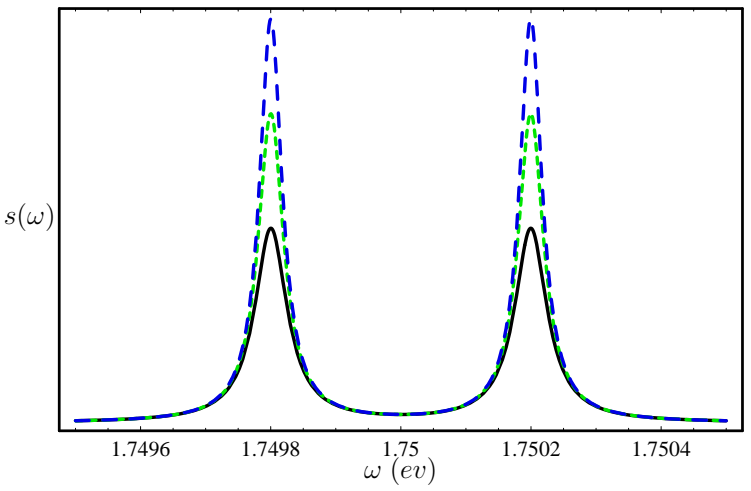

Figure 2. Plots of $S(\omega)$ versus $\omega$. Parameter are choose as $\omega=1.75 \mathrm{eV}$, $\omega_{e x}=1.75 \mathrm{eV}, g=200 \mu \mathrm{eV}, \gamma_{e x}=20 \mu \mathrm{eV}, \gamma_{p h}=40 \mu \mathrm{eV}, n=1$ and $|\alpha|^{2}=9$. In all figures we have $q=1$. Solid line corresponds to case $s=1$. In dotted one we have $s=1.007$ and for dash line $s=1.01$.

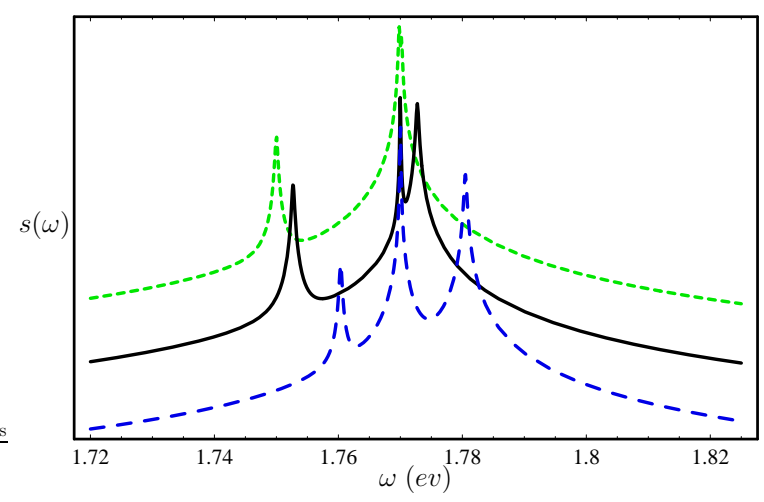

Figure 3. Plots of $S(\omega)$ versus $\omega$. Parameter are choose as $\omega=1.75 \mathrm{eV}$, $\omega_{e x_{1}}=1.75 \mathrm{eV}, \omega_{e x_{2}}=1.77 \mathrm{eV}, g=200 \mu \mathrm{eV}, \gamma_{e x_{1}}=\gamma_{e x_{2}}=200 \mu \mathrm{eV}, \gamma_{p h}=45 \mu \mathrm{eV}$, $n_{1}=1, n_{2}=1$ and $|\alpha|^{2}=9$. Dotted line corresponds to nondeformed case $q_{1}, q_{2}=1$. For solid line $q_{1}, q_{2}=1.04$. In the case of dashed line $q_{1}, q_{2}=1.08$. 


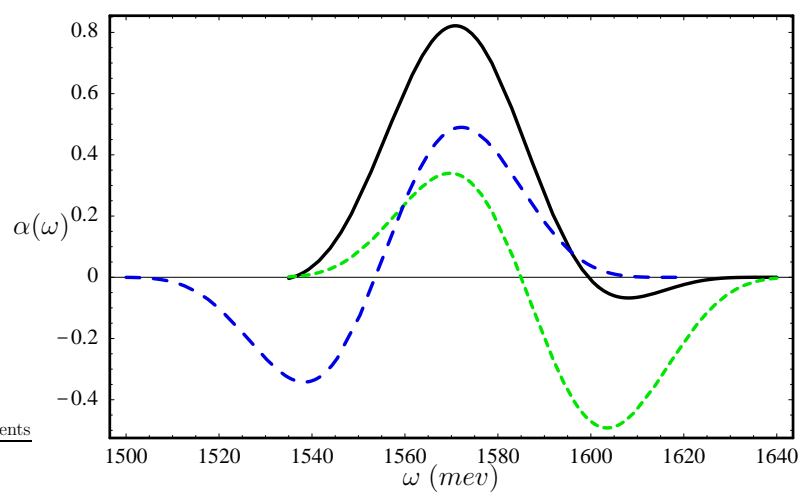

Figure 4. Plots of spectrum absorption versus $\omega$. We consider 1s-exciton and Parameter are choose as $\hbar=e=1, g=200 \mu e v$ and $\omega_{e x}=1574$ mev. Solid plot corresponds to nondeformed case $q=1$. For dotted one $q=1.01$ and in dash one $q=0.99$.

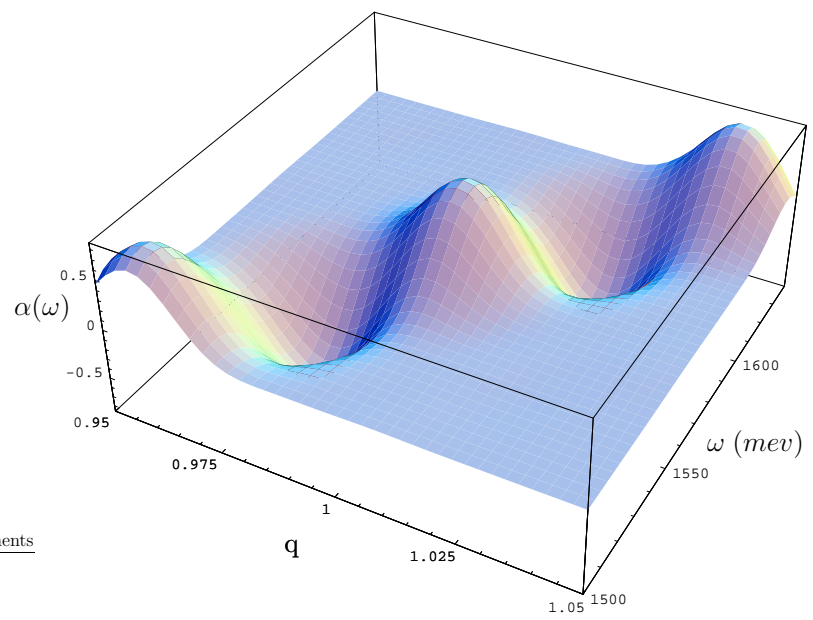

Figure 5. 3D-Plots of spectrum absorption versus $\omega$ and deformation parameter $q$. Physical parameter are the same as Fig.(4).

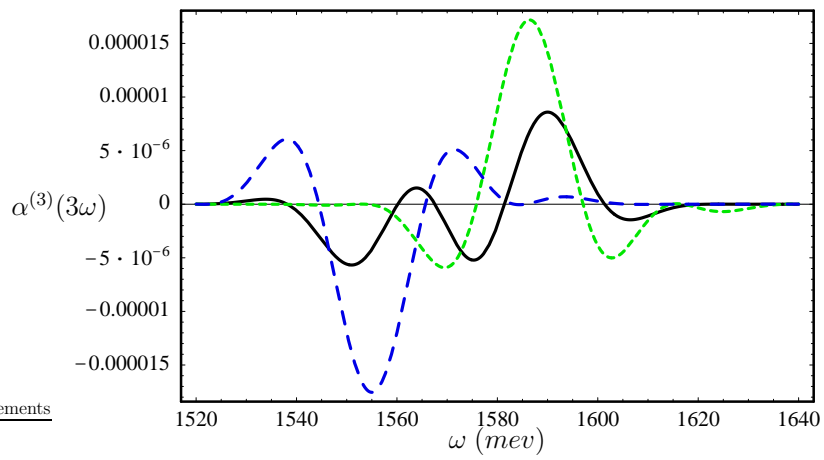

Figure 6. Plots of nonlinear spectrum absorption versus $\omega$. We consider 1s-exciton and Parameters are choose as $\hbar=e=1, g=200 \mu e v$ and $\omega_{e x}=1574$ mev Solid plot corresponds to nondeformed case $q=1$. In dotted plot $q=1.01$. In dash plot $q=0.99$. 